
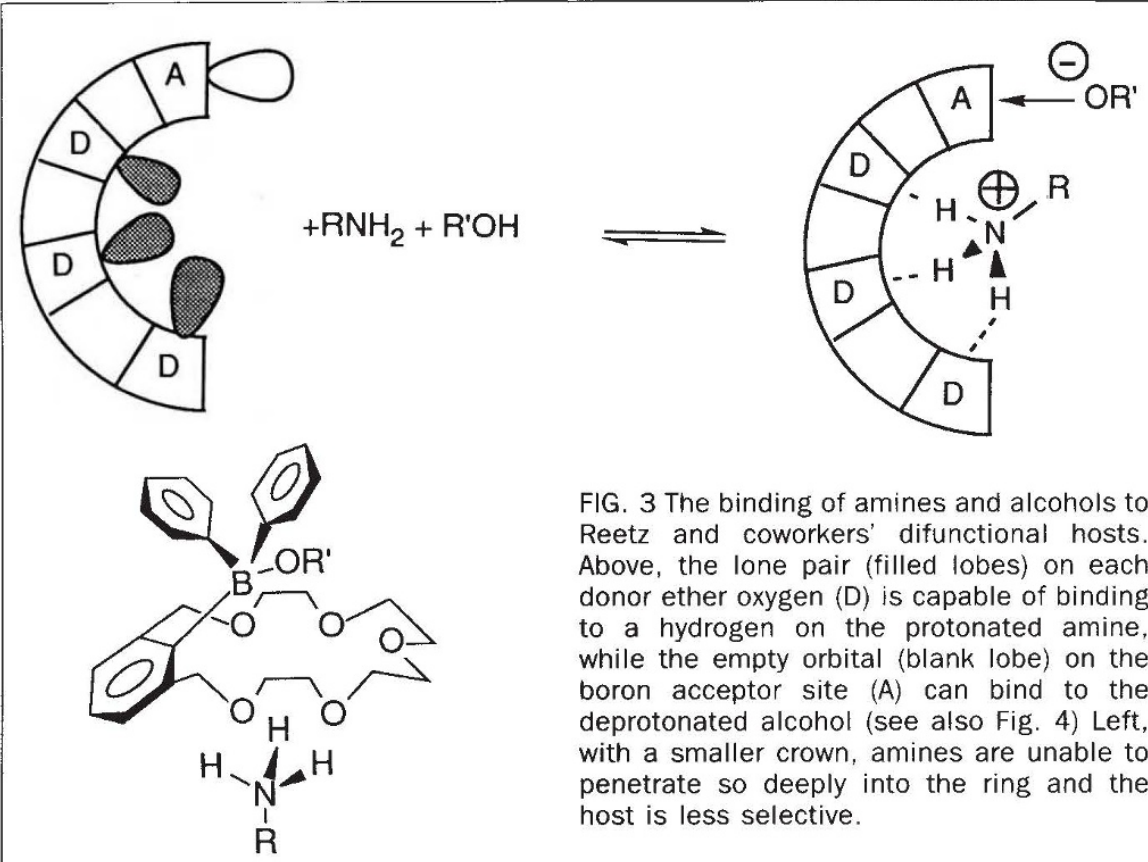

FIG. 3 The binding of amines and alcohols to Reetz and coworkers' difunctional hosts. Above, the lone pair (filled lobes) on each donor ether oxygen (D) is capable of binding to a hydrogen on the protonated amine, while the empty orbital (blank lobe) on the boron acceptor site (A) can bind to the deprotonated alcohol (see also Fig. 4) Left, with a smaller crown, amines are unable to penetrate so deeply into the ring and the host is less selective.

volved the interaction of the difunctional host with a 1:1 mixture of methanol and benzylamine in dichloromethane. An Xray structure of the product shows that there is communication between the guests: the ammonium ion is contained in the cavity of the crown ring and is hydrogen bonded to the oxygens of the crown as well as to that of the methoxy group (which is bound at the boron acceptor site). Competition experiments involving benzylamine and two alcohols

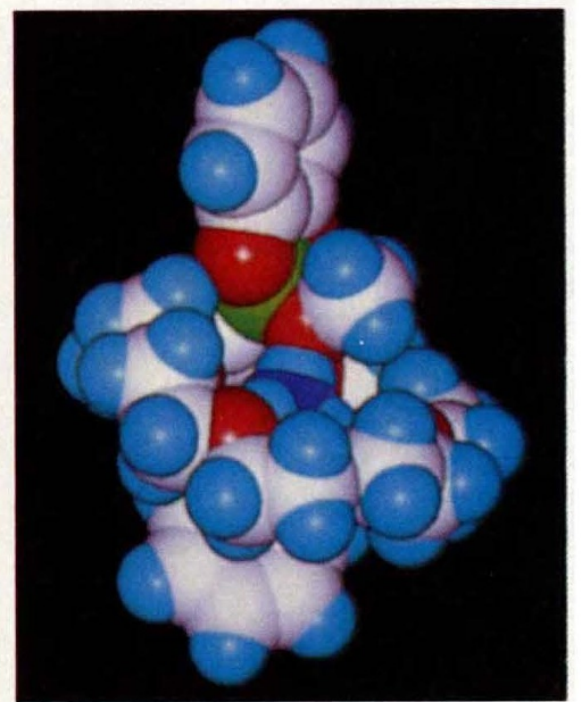

FIG. 4 Space-filling model of Reetz and coworkers' difunctional host bound to methanol and benzylamine. Carbon, white; hydrogen, pale blue; boron, green; oxygen, red; and nitrogen, purple. The methanol (in its methoxy form) is tucked into the fold to the right of the boron. The ammonium cation is tucked into the ether ring just below, its hydrogens bonded to the ether oxygens; the benzyl moiety extends below the ether ring. There is a further hydrogen bond between the ammonium ion and the methoxy oxygen. indicate that selective alcohol binding occurs. For example, methanol binding is favoured over ethanol binding by a ratio of approximately $3: 1$. Such potentially useful molecular recognition appears to be largely controlled by steric factors.

Because the $\mathrm{X}$-ray structure shows incorporation of benzylamine in the crown's cavity, the authors expected that the host might recognize amines selectively based on their size. Again, competition experiments with benzylamine and $\alpha$-methylbenzylamine together with different alcohols show that, in each case, only a benzylamine/alcohol combination was selected.

Similar competition experiments involving a smaller version of the host (incorporating five oxygen atoms in the crown ring, instead of six) resulted in lower amine selectivity. Reetz and coworkers ascribe this to the inability of the respective ammonium groups to penetrate as deeply into this smaller crown (Fig. 3). This latter observation is in accordance with the X-ray structure of the ethanol/benzylamine adduct. But this smaller host yields increased selectivity for methanol; for example, in association with benzylamine, methanol is bound exclusively over ethanol.

The present innovations in receptor design have wider implications. These include the development of new anion sensing devices, new chromatography materials and new boron-containing reagents for use in neutron-capture therapy for the treatment of cancer.

Leonard F. Lindoy is in the Department of Chemistry and Biochemistry, James Cook University, Townsville 4811, Queensland, Australia.

\section{Cries to heaven}

Sound, says the physics syllabus, travels in straight lines. Daedalus disagrees. $\mathrm{He}$ points out that air molecules move in the Earth's gravitational field. They travel not in straight lines, but in parabolic paths like any other projectile. The path of a sound wave, being composed of innumerable molecular trajectories, must be parabolic too.

On this argument, you might expect sound to have the same ballistics as a gun whose shell travelled at the velocity of an air molecule. Its maximum range would be about $25 \mathrm{~km}$, achieved by launching it at $45^{\circ}$ to the horizontal. This isn't quite right. A shell slows up as it climbs, but sound doesn't - its speed depends purely on the local air conditions. This extends its range dramatically. High enough angles of launch should project it hundreds of kilometres.

Its maximum practical range, says Daedalus, is set by the height of the atmosphere. $400 \mathrm{~km}$ up, the mean free path is about $25 \mathrm{~km}$. A sound wave which reached this height would launch a set of molecules on purely ballistic trajectories. They would curve over in a huge collisionless parabola until, falling back into the denser atmosphere below, they launched the sound on its return journey to Earth. This atmospheric 'mirror-ceiling' limits the horizontal range of sound to about $300 \mathrm{~km}$. Gunfire has occasionally been heard over this distance.

Daedalus likes the idea of launching a sound into the sky, and having it boom down from the heavens in a narrow target zone many kilometres away. This novel form of directional communication would be welcomed by unscrupulous advertisers and evangelists seeking to demonstrate celestial approval of their claims. But the required phased-array transmitter, with its banks of highpowered loudspeakers distributed over many hectares, could be better employed in atmospheric research.

For this purpose the transmitter would launch an intense continuous tone. The tone would reach the distant receiving station with a complex and rapidly changing amplitude and phase, reflecting the many vicissitudes of its long stratospheric journey: wind shear, Doppler shifts, fluctuations of temperature and so on. The transmitter and receiver would be linked by at least two sonic paths, one a normal parabola and the other bounced off the atmospheric ceiling. The differences between them would be informative, especially over a range of frequencies. A huge volume of atmosphere could be neatly interrogated from the ground. Weather forecasting might be transformed. Nobody outside the system would be deafened.

David Jones 